\title{
Biometric Authentication using Human Footprint
}

\author{
Kapil Kumar Nagwanshi \\ Rungta College of Engineering \& Technology \\ Kohka-Kurud Road Bhilai \\ CG India
}

\author{
Sipi Dubey \\ Rungta College of Engineering \& Technology \\ Kohka-Kurud Road Bhilai \\ CG India
}

\begin{abstract}
The password based authorization scheme for all available security systems can effortlessly be hacked by the hacker or a malicious user. One might not be able to guarantee that the person who is using the password is authentic or not. Only biometric systems are one which make offered automated authentication. There are very exceptional chances of losing the biometric identity, only if the accident of an individual may persists. Footprint based biometric system has been evaluated so far. In this paper a number of approaches of footprint recognition have been deliberated.
\end{abstract}

\section{General Terms}

Biometric pattern recognition, Image processing.

\section{Keywords}

Biometric footprint recognition, PCA, image reconstruction, filtering, ROI, SOM, HMM.

\section{INTRODUCTION}

\subsection{Biometric System}

Traditional systems for user authentication are based on password. Still most of the financial organization doesn't provide the biometric system for user identification. The virtual keyboard system is available with most of the operating systems and browsers, but then again this is not a very efficient system. As soon as the user get catch by hackers it very easy to transfer his money into hackers account. Nowadays this type of token-based system is the major challenge for the transaction world.

Jain et al [1], describes the definition of biometric as the task of biometric (from Greek bios-life, metron-measure) systems consists of determining the individual identity founded on his or her differentiating physiological and/or behavioral features in the majority of cases both properties are addressed [2]. Whereas the term "biometric" may also refer more generally to the application of mathematics and especially statistics to biological sciences, this work explicitly concentrates on the information technologic aspect focusing on authentication tasks. In order to recognize a person, biometric software extracts a machine-readable representation of physiological and/or behavioral characteristics of that person, called feature vector or template [3], to facilitate score-based matching with stored templates in member databases. From this point of view, biometry is an important pattern recognition research problem [4]. Using hand or footprint-based measurements constitutes one of many different possibilities to realize biometric authentication. The term multimodal in the title implies that multiple evidences of the same identity are provided using not only a single biometric indicator, but merging the information of multiple matchers in order to improve accuracy [5]. Biometric blending is a common method to cope with poor recognition rates or performance of unimodal systems [2]. While most multimodal biometric systems incorporate multiple sensors, this effort functions on optical single-sensor output and may therefore be classified as a blending or fusion method assimilating manifold exemplifications and matching algorithms for the equivalent biometric, according to [6] . Finally, the term recognition in this work refers to both verification (1:1 comparison with a claimed identity template) and identification (1:n comparison to find a matching template, if existing), as is used in respective literature [7] [2].

\subsection{Footprint Recognition system}

Very diminutive literature is available for the footprint recognition system. Very first literature has been published by Nakajima et al [8] authorized the technique for the FootprintBased Personal Recognition. In their method, an input duo of raw footprints is standardized, together in direction and in point for sturdiness image-matching between the input pair of footprints and the pair of recorded footprints. Over and above as added biometric systems, the Euclidean distance between them, the geometric information of the input footprint is used proceeding to the normalization. In the research, the pressure distribution of the footprint was measured with a pressuresensing mat. The recognition rate was found to be $30.45 \%$ without any normalization, and $85.00 \%$ with this method.

One might also be identify by the use of human walking activities as advised by Jung et al [9]. As in the previous methods which are based on human gait, stable, relatively continuing walking data are the crucial conditions for person recognition. In future, these methods are very challenging to accomplish with countless change of walking velocity which may be generated often during real walking. In this literature, they recommend a technique which uses just single-step walking records from mat-type pressure sensor. When a human walk through the pressure sensor, it become quantized COP (Center of Pressure) path, and HMM (Hidden Markov Model) is used to make the likelihood models for user's each foot, besides at that moment, they use HMMs for two feet are joined for improved performance by Levenberg-Marquart learning process, on the other hand in this method gait has been considered, not the human footprint as the biometric authentication.

Wang et al [10] deliberated alternative system grounded on gait investigation in his paper. The dissemination of footprint substantial pressure surface reproduces the performance characteristics and the physiological characteristics of the humanoid figure. Consequently, footprint substantial pressure surface pick-up and depiction is the establishment of footprint biological feature identification. They put forward that footprint heavy pressure surfaces pick-up process based on Gauss curvature and average curvature which can pick-up excellently footprint heavy pressure surfaces (which is the material of curiosity too), and on the basis of this process, the effect of experimentation exhibits that, this description of method is simple and pragmatic. Andreas Uhl and Peter Wild 
[11] explore an approach of foot biometric characteristics which is established on the geometry, the shape and the texture. The Image enhancement and the feature extraction stages highlighting on definite characteristics of the foot geometry, their durability and the uniqueness properties, respectively, are also explained by them. Collectability and universality are the important issues and reflected too. The Gait Recognition is a remarkable signal processing tool for the biometric proof of identity [12]. Such as there is no minutia based pattern matching system, study on gait-based identification by reflection of a person's walking style provides indication that such a system is accurate and is possible to be advanced and used in the forthcoming days. Most of the effort has been conceded out during the time period of 2004-05 is created on gait-based identification.

Kuragano [13] and his colleague provide a novel approach based on gait and footprint analysis. Health care providers in Japan assess the recovery status of patients by detecting a variation in the patient's style of walking. In the first phase of psychoanalysis, the manner of walking is uneven. By way of rehabilitation progresses, the mode of walking of the patient turn into stable state. Consequently, changes in the footprint images of the patients may be identified. The techniques of binarization of a foot print image, noise-reduction, and damage and stretching to smoothening of the edge of the binary image to discover the edge of the footprint image are defined. In addition discrete Fourier transformation has been applied to measure the correspondence of footprint patterns and inverse discrete Fourier transformations to eliminate the high frequency mechanisms in the footprint pattern are described. In the year 2008, a doctoral thesis has been published for single-sensor hand and footprint-based multimodal biometric recognition by Peter Wild [14]. He has developed a system for contemporary humanity, and as it is assumed that no complete biometric modality suitable for all the applications has been established. They arranged all the biometric information from spas, and wellness areas. This novel modality provisions confidentiality interests and still offers underneath accuracy.

Recently a technique for footprint recognition has been evaluated by Ambeth Kumar, and Ramakrishnan [15]. In this research the center of pressure (COP) has been taken out by means of stationary technique from the resized attuned footprint image. COP value is determined using the pixel coordinates $(x, y)$, these values are further trained using Principle Component Analysis (PCA), and then stored in templates. PCA diminishes the great dimensionality of the data space to the smaller fundamental dimensionality of feature space, then and there the Matching algorithm associates the test data based upon the three features using the template and demonstrates whether the user is legitimate or not. From above discussion, it is very clear that for personal footprint based identification there are only two domains available: (i) Gait based system, and (ii) Minutia based system. Additionally, one can use any of the hybrid direction i.e. Gait together with Minutia based personal identification.

\section{PROBLEM IDENTIFICATION}

This paper covers most of the literatures in detail. As per discussion in previous section, very few literatures are available under footprint recognition. They are further classified as gait based system and a minutia based system. Now the problem is that how these patterns can be used for personal identification. Whenever if discussion is going on some pattern recognition problem, and if images are there, then for efficient utilization of images for comparison, one has to operate these images with either DCT or DFT. As the problem domain clearly states that the footprint is the object and recognition is targeted with respect to personal identification. The uniqueness of human footprint motivates towards the development of this system. In the subsequent section, this paper deliberates the steps involved into footprint recognition based on both the techniques. Noise reduction is the major challenge.

\section{SOLUTION METHODOLOGY}

\subsection{Pressure Mat Sensor}

To acquire a footprint image, [8] suggests procedure of a pressure-sensing mat. The pressure distribution of a footprint image (monochrome) is represented as given in eq. (1):

$$
f(x, y):-\frac{X}{2}<x \leq \frac{X}{2},-\frac{Y}{2}<y \leq \frac{Y}{2}
$$

(where $x$ and $y$ are the widths of the mat). Information of the sensor and technique for obtaining the footprint are described in the following section. In this section, we designate a standardization procedure and a recognition method. Consider the following 5-step algorithm (Algo. 3.1) for footprint recognition:

\section{Algorithm 3.1:}

1. Translation gesture of whole image, the center of mass of entire image.

2. Segmentation of footprint (the superior box) and parting of both feet (the inferior boxes).

3. Calculation of the center of foot, + and $\times$ : the center of mass of left and right feet, correspondingly (superior boxes), and translation motion of foot image (inferior boxes).

4. Standardization or Normalization in the direction of footprint images;

5. Recreated or Reconstruction of the whole footprint image.

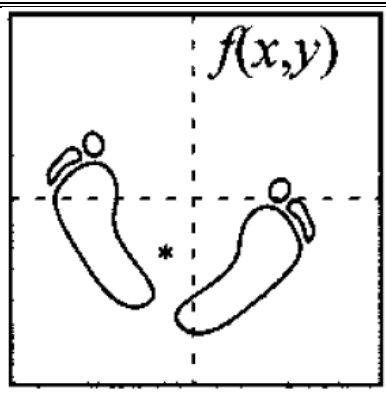

(a). The Acquired Raw Image

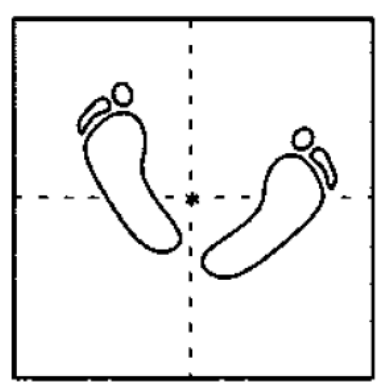

(b). Translation of image 

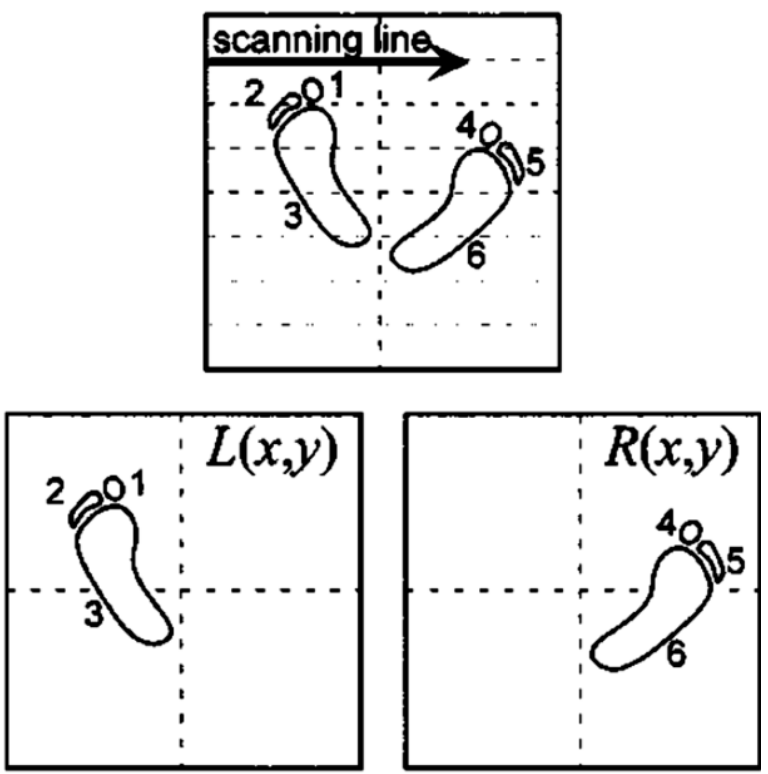

(c). Segmentation of images
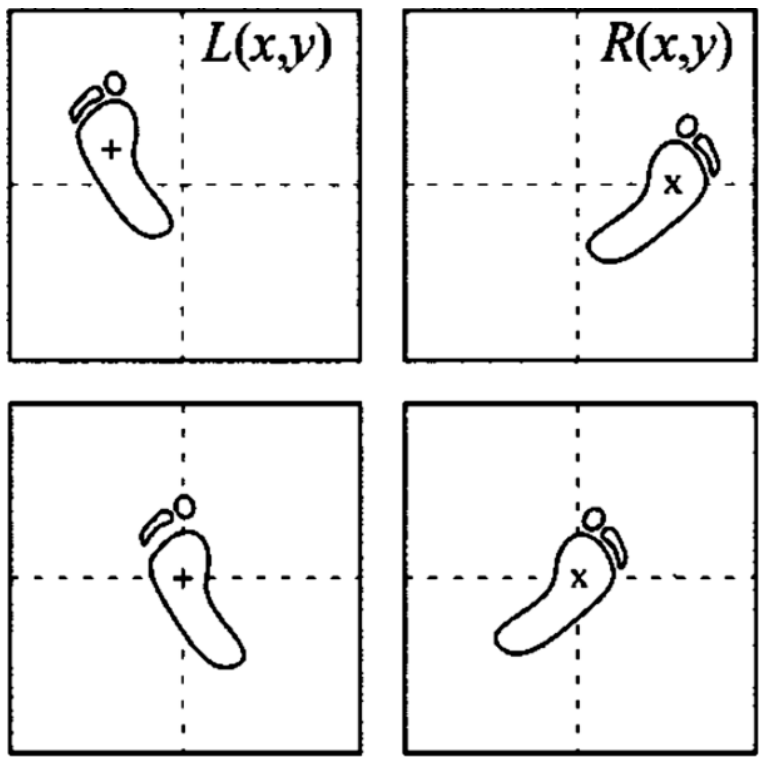

(d). Calculation of the center of foot
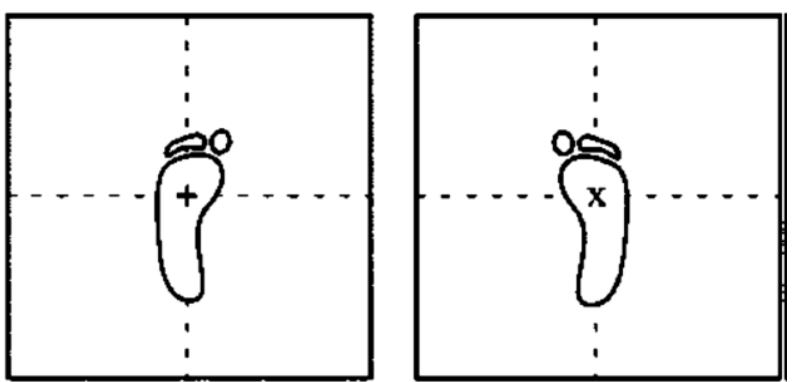

(e). The process of standardization

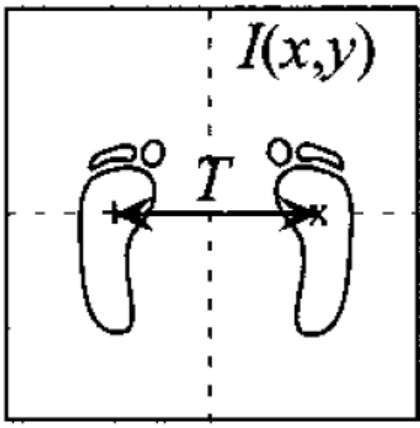

(f). Reconstruction of footprint

Fig 1: The process of footprint recognition [8]

Fig. 1 represents the algorithmic process discussed in Algo. 3.1. On the basis of these algorithmic steps the recognition is going one by one.
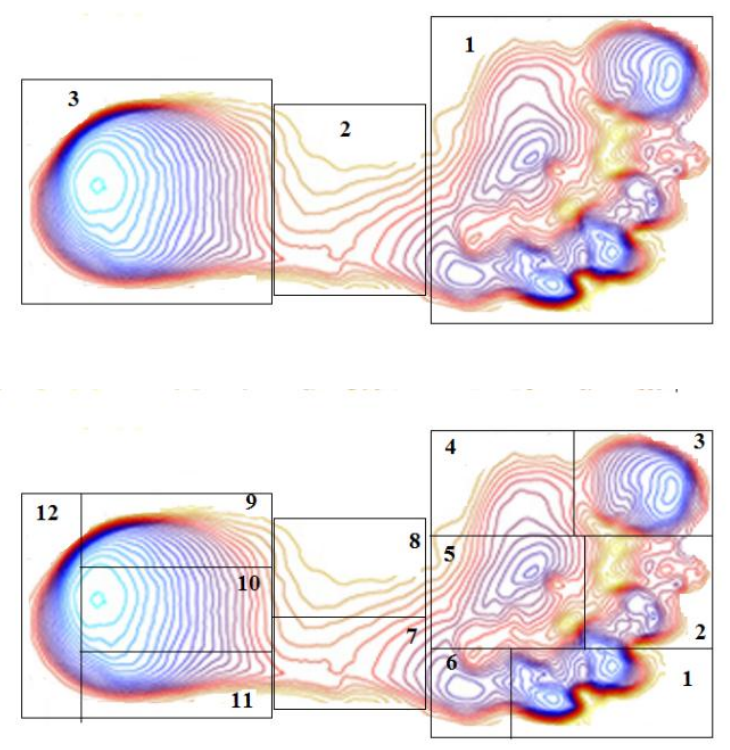

Fig 2: Footprint pressure surface distribution map and Template region

\subsection{Image segmentation}

The influence area in 3-D footprint surface is called as the heavy pressure surface [10]. It exposes individual with the pressure distribution on medium. Since the substantial pressure surfaces are decided by human physical appearances, we can understand the personality recognition through the abstracting of heavy pressure surfaces and analyzing their shape features. The system of public security can determine or deny suspected person after comparing the scene footprint with already some footprint models. Footprint pressure attributed divides the footprint into 3 areas and 12 templates (See Fig. 2).

The arc of the footprint apparent does not move away with position, and direction of the curved surface, and has not anything to do using the parameter scheme of the curved surface. Resulting step performed by Wang et al [10] is the segments footprint on the preliminaries of average curvature and Gauss curvature. 


\subsection{Noise Reduction}

Foot print images are acquired from various sources such as pressure sensor mat, CCD camera or some other type of sensors. This is shown in Figure 1(a). While the captured images have been managed or resized, the noise may be introduced, these white and black spots called salt-and-pepper noise [16]. This has been triggered by the reticulated pattern of the socks worn out by the patient when creating the images. Salt-and-pepper noise involves very high and very low lightintensity. In lieu of such extreme high or low light-intensity, a median filter is operative to average the image. As a result, a median filter be there applied.

\subsection{Binerization}

Edge detection is required for identification of footprint patterns. It is very challenging to notice edges by means of a Laplacian transform, for the reason that, the light-intensity gradient of a gray-scale image [10]. Consequently, the entire image has to be binarized for edge detection. A particular threshold value is not sufficient to binarize the whole image. Thus, the entire image is divided into multiple sections, and threshold values for such section are established autonomously. Kuragano [13], has divide the image into 64 regions. The 64 threshold values for every single region are determined, and the entire image is then binarized.

\subsection{Transformation}

Footprint surface topographies are usually extracted using transform-based method such as Fourier Transform [17] and Discrete Cosine Transform [18]. Despite the fact that using Discrete Cosine Transform, some of the points are neglected leading to an imprecise inference. Also Fourier Transform involves floating-valued signals to integer-valued signals; as a result less accuracy has been obtained. Moreover, Wavelet Transform [19] is also used to extract the texture features of the footprint. In the work presented by Kumar and Ramakrishnan [20], a Sequential modified Haar Wavelet is proposed to find the Modified Haar Energy (MHE) feature. As in section 2 it has previously deliberated the importance of Discrete Fourier transformation to measure the correspondence of foot print patterns. To correct a number of lengths of the foot print pattern boundaries, the total length of the perimeters are rescaled to 1 . The process of pattern identification has been carried out. A carefully worked-out function to be discrete Fourier transformed is established using Figure 3 as described in Algo. 3.2:

\section{Algorithm 3.2:}

1. The rescaled total perimeter length of the foot print pattern is divided by sampling point number $m$.

2. Determine a sample point randomly and allocate it the ordinal number "first". $n p=1$.

3. Estimate the distance $\left[d\left(\frac{1}{m} \times 1\right)\right]$ to the centroid.

4. Determine the succeeding sample point, which is $1 / m$ from the "first" sample point and assign it the ordinal number "second". $(n p=$ 2).

5. Compute the distance $\left[d\left(\frac{1}{m} \times 2\right)\right]$ to the centroid.

6. Evaluate the angle stuck between the lines leading from the centroid to sample points "first" and "second".
7. Move to the next sample point "third" by stepping $1 / \mathrm{m}$ and recap the procedure.

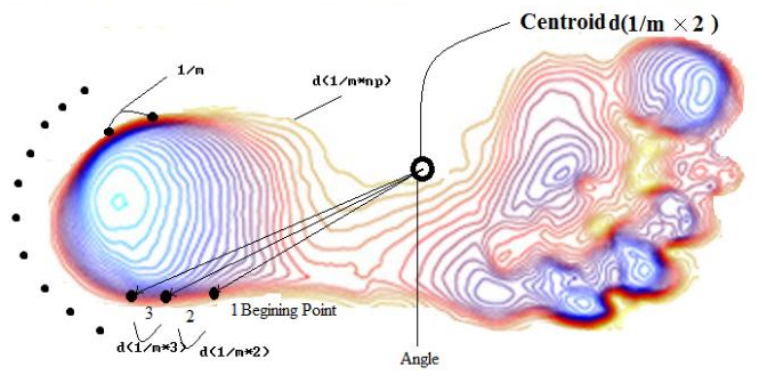

Fig 3: Footprint pattern by DFT [13]

The Haar wavelet coefficients are characterized by decimal numbers. It required eight bytes for storing each of the Haar constants. The division of the subtraction results in y-axis procedure will also diminish the transformation between two adjacent pixels. As a result of these, successive Haar wavelet that maps an integer-valued pixel onto another Integer-valued pixel is advised. Sequential Haar coefficient requires merely two bytes to store each of the extracted constants. The elimination of the division in subtraction results avoids the convention of decimal numbers while preserving the difference between two adjacent pixels. For every image, the image is further divided into $4 \times 4$ blocks. The Modified Haar Energy for each of the block is calculated using eq.(2)

$$
\mathrm{M}_{i, j, k}=\sum_{p=1 ; q=1}^{P Q}\left(C_{p, q}\right)^{2}
$$

where $i$ is the level of decomposition, $j$ is Horizontal, Vertical or Diagonal details, $k$ is the block number from 1 to 16 , $P \times Q$ is the size of the block. The MHE energy feature for every detail constants are organized as in eq.(3)

$$
\mathrm{M}_{i, j}=\left\{\mathrm{M}_{i, j, 1}, \mathrm{M}_{i, j, 2}, \mathrm{M}_{i, j, 3}, \ldots . \mathrm{M}_{i, j, 16}\right\}
$$

As a final point, the Modified Haar Energy (MHE) footprint image is stored in the database.

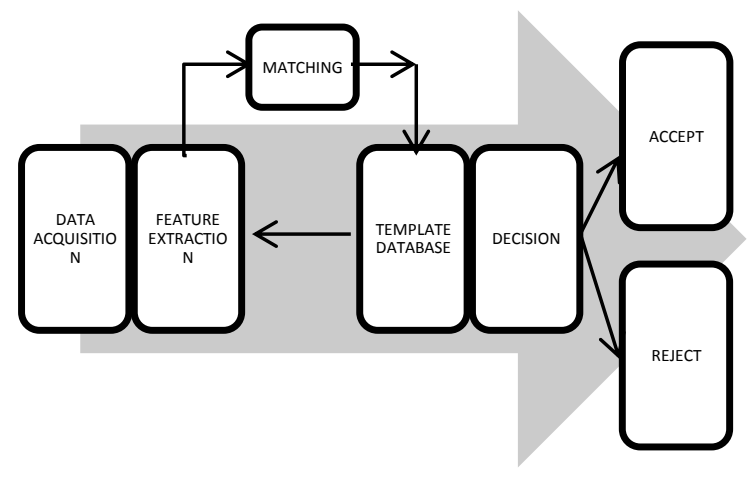

Fig 4: Footprint Recognition Process

\subsection{Matching and Decision module}

To analyze the footprint, two different matching parameters have been used viz. False Acceptance Rate (FAR) and False Rejection Rate (FRR) for the optimistic identification task. Additional extension to a multimodal foot biometric system [4] might be convenient or even necessary meanwhile the 
scalability issues of hand or the foot geometry (features are acknowledged to be not very idiosyncratic and can barely be scaled up for identification out of large population databases) are most prospective to be inherited to the foot biometrics. Fig 4 represents the universal model of footprint recognition system. Recognition Method is also called as Testing. The normalization procedure removes the geometric information of input footprint (raw image). In Testing, normalization is done for the footprint image and then it is compared with the one stored in the database.

\section{RESULT AND DISCUSSION}

In this paper we have omitted all studies before year 2000. It is the fact that most of the publication discusses pattern algorithms significantly. Kohnan [21] did the great work as established a model based on self-organizing maps, a technique for dimensionality reduction. SOM is a kind of neural network algorithm used to cluster the complex data by mapping multi-dimensional data onto a 2-dimensional space, and correlating the mapped 2-dimensional data with each other, on the other hand this technique is not suitable for small and simple data sets which are one dimensional in nature. Weights of correct regions or meaningful spots are increased.

The algorithm based on ART2 has been applied by Chin et al [22]. This technique has been evaluated for automatic threshold selection, and uses the contour shape of the graph created by accumulating distances between all spots of a footprint pattern image. It is slightly difficult to pre-select an initial threshold value since the sizes of feet and strides vary with the species of insects. ART2 based algorithm exhibits good performance in clustering. Kadyrov et al [23] has evaluated the Trace Transform Technique to build a new type of data structure from the segmented images, by defining functions based on parallel trace lines. It is a must to do the tracing in a parallel manner. It will distinguish species of insects to a specified degree.

Again a method based on Neural Network has been developed by Yun et al [24], it recognizes the user with well-trained neural networks. Numerous input nodes in the neural network, causing heavy computation loads, and a significant recognition rate $92 \%$ have been encountered. Smoothing Algorithm developed by Yang et al [25] removes the noise and protects the outlines of footprint image. This is very successful algorithm. Filtered images may have artificial images.

Kumar and Ramakrishnan [15] [26] had compared a number of footprint recognition method. They covered about fifteen distinguished techniques. The Euclidean distance [8] technique is very efficient, and $85 \%$ recognition rate has been observed. Person identification has been done among 10 men using normalized static footprint. The problem with this method is User should make stand-up posture every time. Jung [9] established a technique based on Hidden Markov Model in which Pattern identification has been done among 5 men using position- based quantization of COP (Center of Pressure) from shoe-type pressure sensor. This technique produces $100 \%$ recognition of footprint. Again they applied person identification among 8 men using the dynamic footprint from Mat-type pressure sensor. Kumar [26] get quantized COP trajectory and HMM's for two footprints are combined with Levenberg-Marquart learning method for better performance, but the recognition rate is $64 \%$ which is not equally distributed and not enough high. Table 1 gives the detail of the footprint matching scenario.
Table 1. Matching score of groups A, B, and C

\begin{tabular}{|c|c|c|c|c|}
\hline & A1 & $\mathbf{A 2}$ & A3 & AVERAGE \\
\hline A1 & $95.43 \%$ & $94.12 \%$ & $87.20 \%$ & \multirow{3}{*}{$90.35 \%$} \\
\hline A2 & $88.40 \%$ & $93.60 \%$ & $84.56 \%$ & \\
\hline \multirow[t]{2}{*}{ A3 } & $88.01 \%$ & $89.80 \%$ & $92.05 \%$ & \\
\hline & B1 & B2 & B3 & \\
\hline B1 & $92.44 \%$ & $86.52 \%$ & $84.11 \%$ & \multirow{3}{*}{$88.09 \%$} \\
\hline B2 & $88.32 \%$ & $90.10 \%$ & $86.71 \%$ & \\
\hline \multirow[t]{2}{*}{ B3 } & $87.54 \%$ & $85.97 \%$ & $91.11 \%$ & \\
\hline & $\mathrm{C1}$ & $\mathrm{C2}$ & $\mathrm{C3}$ & \\
\hline C1 & $90.08 \%$ & $87.05 \%$ & $84.76 \%$ & \multirow{3}{*}{$87.71 \%$} \\
\hline $\mathrm{C} 2$ & $87.33 \%$ & $92.03 \%$ & $86.55 \%$ & \\
\hline $\mathrm{C3}$ & $84.11 \%$ & $86.34 \%$ & $91.10 \%$ & \\
\hline
\end{tabular}

\section{CONCLUSION}

This paper provides a brief review of footprint recognition from year 2000 onwards. In result section of these papers, insect's footprint recognition has also been introduced to complete the review. Different algorithm follows different stages of footprint recognition. Mainly footprint recognition is categorized into three stages: image pre-processing, feature extraction and template matching. Image pre-processing is the process used to minimize or reduce the noise from original image. In feature extraction process, the features such as pressure, location and weight are extracted from an image and it improves the performance of the footprint recognition and the input image in matching process. By using all these process, it increases the clarity of the image and high matching score can be obtained. Foot print based authentication bears enough security without drawback of relying on sensitive data demanded by high security applications. Future work includes implementing quality estimation algorithms into a quality based recognition and authentication system to provide an extensive path in this area.

\section{REFERENCES}

[1] A. K. Jain, L. Hong and S. Pankanti., " Biometric identification," Commun. ACM, vol. 43, no. 2, pp. 90-98, 2000 .

[2] D. Maltoni, D. Maio, A. K. Jain and S. Prabhakar, Handbook of Fingerprint Recognition, New York USA: Springer, 2003.

[3] "NSTC Biometrics Glossary," NSTC, 14 September 2006. [Online]. Available: http://www.biometrics.gov/Documents/Glossary.pdf. [Accessed 19 June 2012].

[4] A. K. Jain, S. Pankanti, S. Prabhakar, L. Hong, A. Ross and J. L. Wayman, "Biometrics: A Grand Challenge," in 17th International Conference on Pattern Recognition (ICPR), Cambridge, UK, 2004.

[5] A. A. Ross and A. K. Jain., " Information fusion in biometrics," Pattern Recognition Letters, vol. 24, pp. 21152125, 2003. 
[6] A. K. Jain, A. Ross and S. Prabhakar, "An Introduction to Biometric Recognition.IEEE," IEEE Transactions on Circuits and Systems for Video Technology, vol. 14, no. 1, pp. 4-20, 2004.

[7] R. M. Bolle, J. H. Connell, S. Pankanti, N. K. Ratha and A. W. Senior, Guide to Biometrics, NewYork, USA: Springer, 2004.

[8] K. Nakajima, Y. Mizukami, K. Tanaka and T. Tamura, "Footprint-Based Personal Recognition," IEEE TRANSACTIONS ON BIOMEDICAL ENGINEERING, vol. 47, no. 11, pp. 1534-1537, November 2000.

[9] J.-W. Jung, Z. Bien, S.-W. Lee and T. Sato, "DynamicFootprint based Person Identification using Mat-type Pressure Sensor," in Proceedings of the 2Sa Annual Intemational Conference of the IEEE EMBS, Cancun, Mexico, September 17-21,2003.

[10] W. Wang, X. Ping and Y. Ding, "Footprint Heavy Pressure Surface Pick-up and Description," in IEEE Computer Society-Third International Conference on Image and Graphics (ICIG'04), Hong Kong, China, 18-20 December 2004 .

[11] A. Uhl and P. Wild, "Footprint-based biometric verification," Journal of Electronic Imaging-Society of Photo-Optical Instrumentation Engineers, vol. 17, no. 1, p. 011016, Mar 19, 2008.

[12] N. V. Boulgouris, D. Hatzinakos and K. N. Plataniotis, "Gait Recognition : A challenging signal processing technology for biometric identification," IEEE Signal Processing Magazine, vol. 22, no. 6, pp. 78-90, November 2005.

[13] T. Kuragano, A. Yamaguchi and S. Furukawa, "A Method to Measure Foot Print Similarity for Gait Analysis," in IEEEComputer Society- 2005 International Conference on Computational Intelligence for Modelling, Control and Automation, and International Conference Intelligent Agents, Web Technologies and Internet Commerce (CIMCAIAWIC'05), 2005.

[14] P. WILD and A.-P. M. D. Andreas, "Single-sensor hand and footprint-based multimodal biometric recognition: $\mathrm{A} \mathrm{PhD}$ Thesis," Naturwissenschaftlichen Fakultät der Universität, Salzburg, January 2008.

[15] V. Kumar and M.Ramakrishnan, "Footprint Recognition with COP Using Principle Component Analysis (PCA)," Journal of Computational Information Systems, vol. 8, no. 12, pp. 4939-4950, 2012.
[16] R. C. Gonzalez and R. E. Woods, Digital Image Processing, 3rd Edition, New Delhi: Pearson, 2008.

[17] W. Li, D. Zhang and Z. Xu, "Palmprint Identification By Fourier transform," Intl.Journal of Pattern Recognition and Artifical Intelligence, vol. 16, no. 4, pp. 417-432, 2002.

[18] X.-Y. Jing and D. Zhang, "A Face and Palmprint Recognition Approach Based on Discriminant DCT Feature Extraction," IEEE Transaction on on Systems, Man. and Cybernetics -Part B:Cybernetics, vol. 34, no. 6, pp. 2405 2415., December 2004.

[19] K.-Q. W. a. D. Z. Xiang-Qian Wu, "Wavelet Based Palm Print Recognition," in First International Conference on Machine Learning and Cybernetics, November 2002.

[20] V. D. A. Kumar and M. Ramakrishnan, "Footprint Recognition using Modified Sequential Haar Energy Transform (MSHET)," IJCSI International Journal of Computer Science Issues \{ISSN (Online): 1694-0784\}, vol. 7, no. 3, pp. 47-51, 2003.

[21] T. Kohonen, Self-Organizing Maps, 3e, Springer Series in Information Sciences, 2001

[22] B. Shin, E. Cha, Y. Woo and R. Klette, "Segmentation of Scanned Insect Footprints Using ART2 for Threshold Selection," LNCS , Springer-Verlag, vol. 4872, pp. 311-320, 2007.

[23] A. Kadyrov and M. Petrou, "The Trace Transform and Its Applications," IEEE Transactions on Pattern Analysis and Machine Intelligence, vol. 23, no. 8, pp. 811-828, 2007.

[24] J. Yun, S. Lee, W.Woo and J. Ryu, "The user identification system using walking pattern over the ubiFloor," in International Conference on Computational Intelligence and Security, Xi'an, China, 2005.

[25] S. Yang, C.-r. Wang and X.-y. Wang, "Smoothing Algorithm Based on Multi-Scale Morphological Reconstruction for Footprint Image," in Second International Conference on Innovative Computing, Informatio and Control (ICICIC 2007), Kumamoto City International Center, Kumamoto, Japan, September 2007.

[26] V. A. Kumar and M. Ramakrishnan, "Legacy of Footprints Recognition- A Review," International Journal of Computer Applications (0975 - 8887), vol. 35, no. 11, pp. 9-16, December 2011. 